\title{
Ergonomía digital y su influencia en el aprovechamiento académico de las clases virtuales en enfermería
}

\section{Digital ergonomics and its influence on academic achievement in online classes on Nursing}

DOI: https://doi.org/10.32870/dse.v0i24.1055

\author{
Víctor del Carmen Avendaño Porras* \\ Mariano Raymundo Hernández Hernández**
}

\begin{abstract}
Resumen
Las condiciones sanitarias actuales por la pandemia de Covid-19, han generado que las clases presenciales del nivel superior en México se impartan por medio de plataformas virtuales sincrónicas y asincrónicas, utilizando dispositivos móviles y computacionales desde el hogar; esto conlleva que los estudiantes adecúen su entorno virtual de aprendizaje y adopten posturas acordes a la ergonomía digital al hacer uso del mobiliario que disponen para las clases virtuales. La presente investigación es de corte transversal y cuantitativo, se diseñó y validó un instrumento ex profeso de 36 ítems que se aplicó a 225 estudiantes de la Licenciatura en Enfermería de la Universidad Mesoamericana, en donde se encontró que $92 \%$ no cuentan con un ambiente virtual de aprendizaje idóneo al recibir sus clases en cuartos individuales, y que $20 \%$ de los alumnos coloca a una distancia ideal su dispositivo móvil y equipo de cómputo con una postura ideal. De esta manera, se concluye que se tienen que generar procesos de capacitación al alumnado para que, en la medida de sus posibilidades, adecúen su entorno virtual de aprendizaje (EVA) y limitar los daños que pueden generar las posturas inadecuadas.
\end{abstract}

Palabras clave: pandemia - universidad - educación virtual - ambiente virtual de aprendizaje - ergonomía.

\begin{abstract}
The current sanitary conditions of COVID-19 have forced higher education in Mexico to be taught through synchronous and asynchronous online platforms, using mobile and computer devices at home. Students have had to adapt to their virtual learning environment and adopt positions in accordance with digital ergonomics when using the furniture available for online classes. This cross-sectional and quantitative research designed and validated ad hoc instrument of 36 items applied to 225 students of the Bachelor of
\end{abstract}

* Doctor en Educación. SNI 1. Coordinador de la Cátedra Internacional de Interculturalidad y Pensamiento Crítico de la Universidad de la Serena, Chile. Profesor-investigador de la Universidad Mesoamericana. México.victor.avendano@unimeso.edu.mx

** Estudiante del Doctorado en Educación. Profesor-investigador de la Universidad Mesoamericana. México. enfrayuma@gmail.com 
Nursing at the Mesoamerican University. We found that $92 \%$ do not have an adequate online learning environment to take their classes in individual rooms, and that $20 \%$ of the students place their mobile device and computer equipment at an ideal distance with an ideal posture. We concluded that student training processes have to be generated so that they can adjust their virtual learning environment (EVA) as well as possible and limit the damage that inappropriate postures can generate.

Keywords: pandemic - university - virtual education - virtual learning environment - ergonomics.

\section{Introducción}

La educación superior en México marca la pauta para el desarrollo y transformación nacional en los sectores económico, social, político, cultural y de salud pública, al generar y capacitar a los recursos humanos para su desempeño óptimo en los diferentes sectores productivos y de servicios que el país posee (Laredo, 2020).

El contexto de la globalización y la era digital exigen a los países en vías de desarrollo incursionar en la innovación de la educación para adecuarse a los nuevos métodos de enseñanza por medio de plataformas virtuales y acercarse a un mayor número de jóvenes, mejorando las oportunidades de acceso a la formación universitaria a la población que se encuentra en los medios rurales y urbanos (Moreno, 2011).

De acuerdo con los datos presentados por Calderón (2020), la capacidad de respuesta del Sistema Educativo Nacional en la vertiente del nivel superior cuenta en la actualidad con 5,716 universidades, de las cuales 2,311 pertenecen al sector público, con una matrícula estudiantil de casi 3 millones, y 3,405 universidades pertenecen al sector privado, con una matrícula estudiantil de más de un millón, que se encuentran en formación en una de las mil carreras que se ofrecen en todo el territorio mexicano.

La Secretaría de Educación Pública ofrece también datos relevantes acerca de la cobertura educativa del nivel superior, ya que del total de aspirantes de nuevo ingreso del país, sólo 31\% logra ingresar a una universidad pública. Con respecto al tránsito escolar, se tiene una media nacional crítica, ya que de cada 100 alumnos que inician la educación primaria sólo 27 logran culminar una carrera universitaria; en el caso de Chiapas sólo 12 logran este objetivo. Es claro que se tienen brechas amplias con respecto a esta temática al ser un estado con índices de desarrollo humano y económico bajos; con este indicador se manifiesta el marcado rezago educativo ante la comparativa nacional (Mendoza, 2018).

Una de las áreas de formación que representa un eje principal para la salud pública de México es la enfermería, que se imparte como un programa de licenciatura en universidades públicas y privadas del país para coadyuvar a la cobertura de la demanda de servicios de salud de la población (Luengo, 2016). La formación que se tiene en esta carrera en nuestro país es ejemplo en América Latina, esto lo pone de manifiesto Salcedo (2017) en un estudio sobre la 
enseñanza de la enfermería en México, destacando la alta complejidad formativa que estipulan los programas de estudio del país.

La complejidad de la época actual, y ante la pandemia provocada por el Covid-19, ha hecho que las escuelas de enfermería incursionen en la modalidad de trabajo virtual de manera rápida, para poder dar continuidad a las actividades académicas de los programas de estudio y evitar la pérdida de semestres completos. Según estudios realizados por Lara (2020), las instituciones formadoras de enfermería han podido adaptarse de manera satisfactoria a las plataformas virtuales, pero reconoce que esta situación dejó a la vista pública las brechas existentes en el ámbito económico, educativo, innovación y tecnología de las universidades en general, por ende, Torres (2002) reconoce que se tiene que trabajar de manera más ardua en las estructuras del Sistema Educativo Nacional para favorecer el aumento de la capacidad educativa y la mejora e innovación de la tecnología educativa.

Ante esta situación acerca de la educación virtual, las brechas educativas y tecnológicas, se han manifestado las limitantes y dificultades de acceso equitativo a los estudiantes del nivel superior, marcado por estratos sociales y económicos, dispersión poblacional, diversidad étnica, el acceso a la tecnología educativa, las computadoras y la conexión a internet, que son las constantes problemáticas que enfrentan de manera frecuente (Lloyd, 2020).

Estos nuevos escenarios de aprendizaje para la educación superior en enfermería en su modalidad no presencial se conceptualizan como Ambientes Virtuales de Aprendizaje (AVA), en donde la institución educativa genera y propicia ambientes y entornos de clases en plataformas digitales para las clases sincrónicas y asincrónicas y, a la vez, para el estudiante implica la labor de adecuar su espacio para recibir clases en las inmediaciones de su vivienda.

La entrega de conocimientos en tiempos de pandemia se ha apoyado en los AVA, y la mayor parte de las universidades han adecuado plataformas virtuales por medio de software educativos, como los paquetes de e-learning, Moodle, cursos masivos MOOC, para que los estudiantes tengan espacio de interacción y desarrollo de los contenidos de los programas educativos.

Por su parte, Zurita (2020) destaca que los docentes universitarios están obligados a desarrollar habilidades en el manejo de las plataformas virtuales para que puedan trabajar de manera eficiente y adecuada, acercando a los estudiantes a los mejores contenidos posibles para el aprovechamiento de cada una de las clases o actividades que se cargan en las aulas virtuales (Sánchez, 2016). De igual manera, el docente usa particularmente salas de conferencias virtuales para las clases sincrónicas y esto le exige mayor preparación y actualización para el manejo de plataformas durante las sesiones en línea; Zoom, Google Meet, Facebook, Jitsi, Blue y videollamadas, que han sido las herramientas más comunes y de fácil acceso para el docente y los estudiantes, pero exige de este último herramientas tecnológicas para su ingreso, como computadoras de escritorio o portátiles, tabletas y teléfonos móviles (Fernández, 2017). 
La adecuación del Entorno Virtual de Aprendizaje en el hogar de los estudiantes universitarios demanda un espacio físico adecuado para poder llevar cada una de sus clases virtuales o el desarrollo de actividades asincrónicas en las plataformas educativas. Son escasas las investigaciones acerca de cuáles deben ser las condiciones del espacio físico educativo en el hogar, por lo que existe un desconocimiento sobre qué factores pueden estar propiciando o limitando el aprovechamiento de las actividades académicas virtuales y si decantan en un aprendizaje significativo para los estudiantes (Rodríguez, 2015).

Se han manifestado en los estudiantes afecciones posturales y de salud al no contar con el conocimiento de la ergonomía digital para adecuar el espacio educativo en el hogar, lo cual ha propiciado la aparición de dolor muscular localizado en el cuello, espalda, brazos y dedos al estar trabajando en las diferentes plataformas virtuales, limitando con esto el aprovechamiento ideal de los contenidos de las asignaturas que cursan a lo largo de cada jornada académica diaria. López (2020) menciona que las alteraciones más frecuentes de la mala postura en el ámbito educativo son las siguientes: $24.9 \%$ de isquiosurales cortos, $18.7 \%$ de hiperlordosis, $9.3 \%$ de escoliosis y $6.4 \%$ de hipercifosis, por esto se debe comenzar a investigar cuáles son las características ideales de los espacios de aprendizaje en los hogares de estudiantes universitarios, para prevenir estas complicaciones que alteran la salud y limitan la adaptación adecuada a las plataformas virtuales en la educación (Gómez, 2019).

Resulta difícil determinar el aprovechamiento académico y la adecuación del entorno virtual de aprendizaje, así como el apego a la ergonomía digital en las clases virtuales de estudiantes de nivel superior, al ser escasos los estudios sobre esta temática que nos brinden un panorama general de los resultados obtenidos en las clases virtuales, aunque Rivero (2008) menciona que esta modalidad de estudio genera un valor agregado a la formación al utilizar elementos de evaluaciones dinámicas en línea, pizarrones multimedia, videos y contenido diverso de internet, que permiten un aprovechamiento mayor y mejoran los resultados del rendimiento escolar.

Para Chiecher (2019), la educación en línea permite amplias posibilidades de desarrollo individual del alumnado al utilizar sus habilidades y destrezas dentro de las plataformas digitales en los dispositivos de conexión a la aulas virtuales; no obstante, Granados (2019) estima que se presenta el riesgo de tener bajo rendimiento académico dado que existen distractores habituales como el ruido del entorno, las notificaciones de las redes sociales, mensajes y llamadas en el teléfono celular, la facilidad de apertura de otras páginas ajenas durante las clases y la adopción de posturas inadecuadas (López, 2020).

La virtualidad de la educación superior se ha extendido a todas las universidades de la república mexicana, incluyendo las instituciones del estado de Chiapas, en donde se trabaja también de manera virtual para dar seguimiento regular de los ciclos educativos que comprenden los semestres de cada área formativa. En el caso de las escuelas de enfermería de régimen particular, 
como lo es la Escuela de Enfermería de la Universidad Mesoamericana (UNIMESO), se encuentran inmersas en este esfuerzo de la educación virtual, para lo cual han habilitado espacios para la interacción sincrónica por medio de plataformas de videoconferencias para el abordaje de cada una de las clases programadas en cada semana de actividades, y espacios asincrónicos como la plataforma de UNIMESO Virtual, que ofrece a estudiantes y docentes la posibilidad de interactuar fuera de las salas de conferencias para la entrega y recepción de actividades complementarias, facilitando con esto el desarrollo de los programas y contenidos académicos.

Los estudiantes de enfermería de la UNIMESO se han integrado de manera adecuada, a pesar de las limitaciones tecnológicas y de acceso a internet que tienen los municipios a los cuales pertenecen; a ello se añade también la poca información sobre los espacios adecuados de estudio virtual en el hogar y el uso de los dispositivos tecnológicos que utilizan para recibir las clases en línea, los cuales implican factores de distracción, cansancio y disconfort en las actividades académicas diarias.

Por ello, el objetivo de esta investigación es conocer cuáles son las características del entorno virtual de aprendizaje de los estudiantes de la Escuela de Enfermería de la Universidad Mesoamericana, para generar alternativas en la mejora académica y rendimiento escolar.

\section{Metodología}

El presente trabajo de investigación es de corte transversal y cuantitativo; tiene el propósito de identificar las características de la adecuación del Entorno Virtual de Aprendizaje y la ergonomía digital en los hogares de estudiantes universitarios que cursan la Licenciatura en Enfermería en la Universidad Mesoamericana de San Cristóbal de Las Casas, Chiapas.

Los datos se obtuvieron de manera virtual debido a las actuales restricciones de acceso a las instituciones educativas a causa de la pandemia de Covid-19, e incluyó 225 estudiantes que cursan desde el primero hasta el octavo semestre, de los cuales 158 son mujeres y 67 hombres. También es importante destacar que del total de alumnos, 32 pertenecen a la etnia tseltal, 33 son tsotsiles, 3 choles y 1 zoque, que nutren a la universidad con la multiculturalidad del estado.

La recolección de datos se realiza mediante un instrumento de medición diseñado y elaborado de acuerdo a las necesidades de esta investigación; fue sometido a un proceso de validación con un grupo de seis expertos en ambientes virtuales de aprendizaje y herramientas digitales que participaron en un grupo focal para la revisión minuciosa de cada ítem. De igual manera, se realizaron las pruebas de confiabilidad en el software de SPSS, para tener de forma objetiva la identificación de características del entorno virtual de aprendizaje y la ergonomía digital en el hogar de los estudiantes.

Como ya se mencionó, en el proceso de validación se utilizó el peritaje de seis jueces expertos en ambientes virtuales de aprendizaje, quienes calcularon la validez de contenido mediante la obtención del coeficiente $\mathrm{V}$ de Aiken. Las dimensiones abordadas en el instrumento fueron: 
datos generales, herramientas tecnológicas de aprendizaje, entorno virtual de aprendizaje y ergonomía digital. De los 45 ítems originales, nueve fueron eliminados por no obtener valores suficientemente altos para mantenerse; de tal forma que el instrumento final quedó con 36 ítems. Por otra parte, para conocer la fiabilidad, se realizó una prueba piloto del instrumento a un grupo de siete personas y se analizó la consistencia interna por el método de Alfa de Cronbach, resultando 0.813 ( $p<0.01$ ); por lo que el instrumento reúne suficientes propiedades como para ser considerado una herramienta válida y fiable.

En la tabla 1 se puede visualizar los componentes del instrumento de medición.

Tabla 1. Instrumento de medición

\begin{tabular}{|c|c|c|}
\hline Dimensión & Tipo de pregunta & Preguntas \\
\hline Dimensión 1: datos generales. & $\begin{array}{l}3 \text { dicotómicas. } \\
3 \text { de opción múltiple. }\end{array}$ & $\begin{array}{l}\text { Edad. } \\
\text { Sexo. } \\
\text { Semestre que cursa. } \\
\text { Modalidad actual de clases. } \\
\text { Pertenencia a un grupo étnico. } \\
\text { A que grupo étnico. }\end{array}$ \\
\hline $\begin{array}{l}\text { Dimensión 2: herramientas tec- } \\
\text { nológicas de aprendizaje }\end{array}$ & 4 de opción múltiple. & $\begin{array}{l}\text { Dispositivo usado para las clases. } \\
\text { Memoria RAM del dispositivo. } \\
\text { Tipo de conexión a internet. } \\
\text { Empresa proveedora de internet. }\end{array}$ \\
\hline $\begin{array}{l}\text { Dimensión 3: entorno virtual de } \\
\text { aprendizaje }\end{array}$ & $\begin{array}{l}5 \text { de opción múltiple. } \\
1 \text { dicotómica. } \\
8 \text { en escala de Likert. }\end{array}$ & $\begin{array}{l}\text { Espacio usado para las clases virtua- } \\
\text { les. } \\
\text { Tipo de escritorio. } \\
\text { Tipo de asiento. } \\
\text { Entorno de estudio (iluminación, rui- } \\
\text { do, ejercicios, confort, conectividad, } \\
\text { hidratación). } \\
\text { Plataforma de videoconferencia. } \\
\text { Rendimiento escolar. } \\
\text { Horas de conexión al día. } \\
\text { Calificaciones. }\end{array}$ \\
\hline Dimensión 4: ergonomía digital & $\begin{array}{l}1 \text { dicotómica } \\
3 \text { de opción múltiple. } \\
8 \text { en la escala de Likert. }\end{array}$ & $\begin{array}{l}\text { Postura. } \\
\text { Distancia de visualización del teléfo- } \\
\text { no, tablet, computadora. } \\
\text { Seguridad e higiene: limpieza del dis- } \\
\text { positivo, uso de audífonos, ajuste de } \\
\text { brillo, lavado de manos, notificaciones } \\
\text { de redes sociales. }\end{array}$ \\
\hline
\end{tabular}

Nota: Se aprecia en la tabla la construcción del instrumento de medición, sus dimensiones, tipos de ítems y las preguntas generales aplicadas a los estudiantes.

El instrumento de recolección de datos se aplicó por medio de la plataforma de Classroom a cada grupo de estudiantes, mencionando el objetivo de la aplicación para que tuvieran cono- 
cimiento del proceso de investigación que se estaba realizando. La aplicación de instrumento se realizó del 2 al 9 de febrero de 2021.

Para el procesamiento de datos se generó un libro de codificaciones de los resultados obtenidos de la aplicación del instrumento de medición para tener un panorama general de resultados.

La interpretación de los resultados se presenta con gráficas de barras y de pastel, con datos numéricos para hacer posible hacer la correlación del discurso interpretativo y comparativo para determinar las características del ambiente virtual del aprendizaje en el hogar de cada uno de los estudiantes.

\section{Resultados}

En las siguientes gráficas se puede apreciar que los datos recabados en el proceso de investigación sobre el entorno virtual de aprendizaje y la aplicación de la ergonomía digital de los alumnos de la Escuela de Enfermería de la Universidad Mesoamericana son poco adecuados para las necesidades educativas que demanda el cursar una carrera de manera virtual dadas las restricciones sanitarias del Covid-19.

El grupo de muestra consta de 225 estudiantes inscritos en los 8 semestres de la Licenciatura en Enfermería; $70 \%$ son mujeres y $30 \%$ son hombres. Con respecto a su edad, $39 \%$ están en el rango entre 15 y 20 años; 54\% entre 21 y 25 años; $4 \%$ entre 26 y 30 años, y $1 \%$ entre 31 y 35 años. El total de estudiantes manifiesta estar cursando de manera virtual sus diversas clases del programa de estudios.

En los resultados relacionados con las herramientas tecnológicas de aprendizaje se detaIlan los dispositivos utilizados para recibir las clases virtuales por parte de los estudiantes: 113 cuentan con una laptop, 8 tienen computadoras de escritorio, 99 usan sus teléfonos celulares y 5 estudiantes lo hacen en tabletas electrónicas (figura 1).

1 De manera general, los porcentajes que se presentan en adelante se redondean en números enteros eliminando los decimales con el propósito de abreviar. 
Figura 1. Porcentajes de estudiantes por tipo de dispositivo

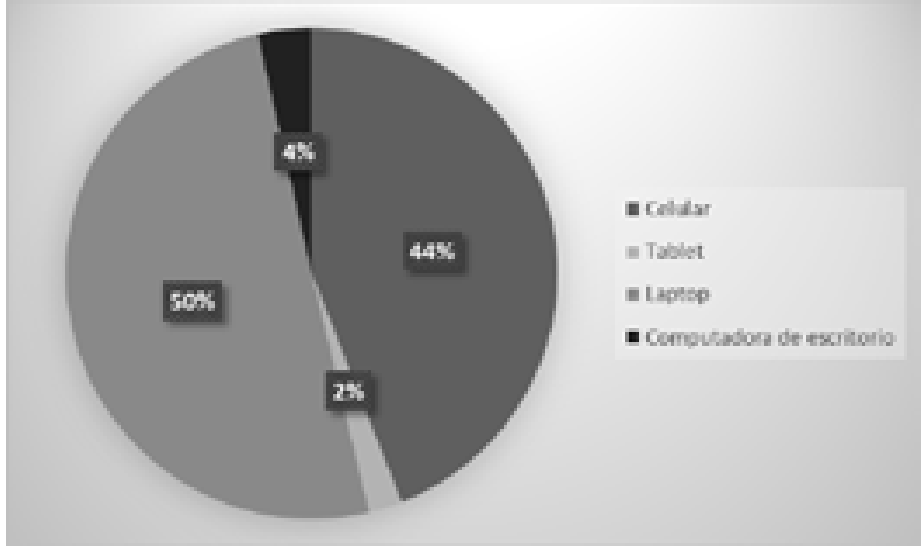

Nota: se aprecia en esta figura el porcentaje de estudiantes por cada tipo de dispositivo para la conexión a las clases virtuales, destacando el uso de celulares y laptop como las principales.

En torno a la capacidad de la memoria RAM, como parámetro para denotar la velocidad de los equipos utilizados en las clases y la resistencia para trabajar con los programas de plataformas virtuales y plataformas de videoconferencias: las computadoras de 11 alumnos cuentan con una capacidad de memoria RAM de 1 GB; 21 con 2 GB; 16 con 3 GB; 63 con 4 GB; 6 con 5 GB; 14 con 6 GB; 2 con 7 GB; 41 con 8 GB; y 51 con más de 8 GB. Esto refleja que 111 alumnos cuentan con dispositivos con un rango de 1 a 4 GB de velocidad, 63 con un rango de 5 a 8 GB y 51 superando los $8 \mathrm{~GB}$.

El servicio de conexión en línea que disponen para el trabajo virtual es el siguiente: $58 \%$ refiere tener internet inalámbrico; $5 \%$ internet satelital; $12 \%$ datos móviles, $16 \%$ con conexión de ethernet; y $5 \%$ no cuenta con ningún tipo de servicio de internet. El suministro de este servicio lo reciben de las siguientes empresas: $16 \%$ Megacable; 39\% Infinitum de Telmex: $21 \%$ Telcel; $1 \%$ AT\&T; y $21 \%$ otra empresa (figura 2).

Figura 2. Cantidad de estudiantes por tipos de servicios utilizados para las clases virtuales

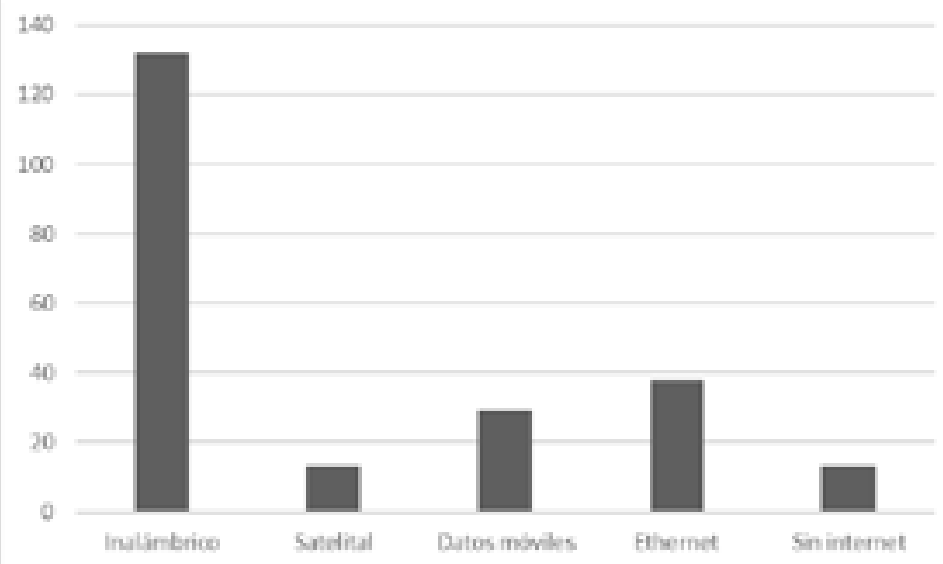

Nota: se aprecia que la mayoría de los estudiantes utilizan conexión vía wifi para el ingreso a sus clases virtuales, seguido del ethernet y datos móviles. 
Con respecto al entorno virtual de aprendizaje, se puede apreciar que $52 \%$ de los estudiantes encuestados recibe sus clases en su cuarto individual, $23 \%$ en la sala, $0.9 \%$ en el patio, $6 \%$ en el comedor, $3 \%$ en la cocina, $3 \%$ mencionan que se trasladan a la casa de un familiar para poder conectarse a sus clases, $2 \%$ se traslada a un ciber público y sólo $8 \%$ adecuó un espacio propio para recibir las clases virtuales. Estos datos representan una gran disparidad en torno a la posibilidad de creación de ambientes de aprendizaje, por lo que en general los espacios de que disponen no son propicios para la actividad educativa, además de que tienen distractores que entorpecen la concentración en las actividades académicas (figura 3).

Figura 3. Cantidad de estudiantes por tipos de espacios destinados para las clases virtuales en el hogar

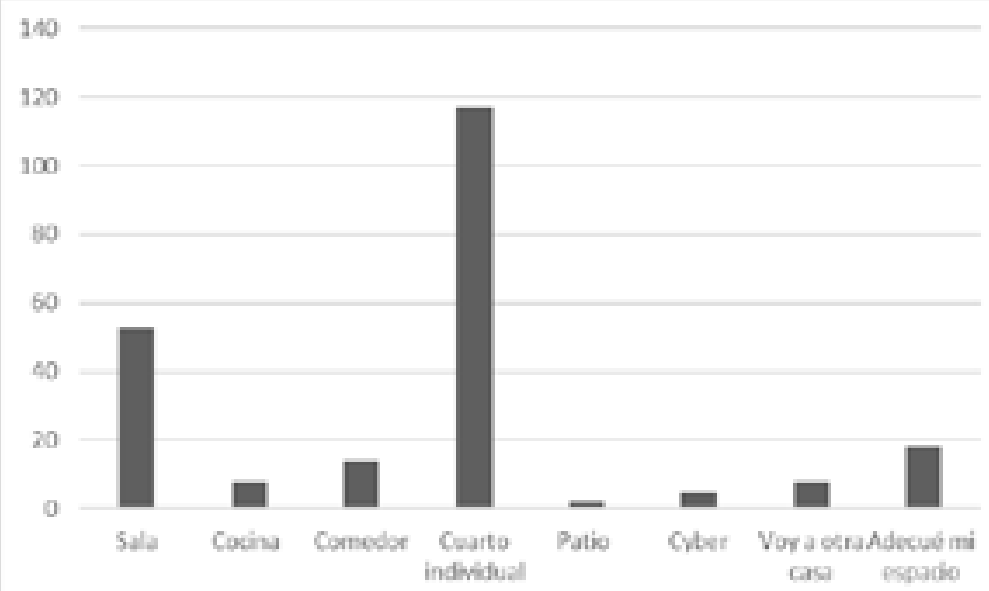

Nota: se aprecia que la mayoría de los estudiantes utilizan su cuarto individual y la sala de sus hogares para recibir las clases virtuales, y un número muy reducido adecuó su propio espacio de manera ideal.

En cuestiones de la ergonomía digital y el mobiliario utilizado para el espacio de trabajo: 90\% de los estudiantes refieren tener como escritorio mesas de madera que tenían previamente en sus hogares, $2 \%$ utilizan una mesa de cristal, y sólo $7.6 \%$ cuenta con un escritorio en forma. En el caso de los asientos utilizados para las clases, 75\% destina una silla de madera, $12 \%$ utilizan sillones, $0.4 \%$ reposet, $3 \%$ bancas de madera, $1 \%$ utiliza su cama como asiento, y sólo $6.7 \%$ cuenta con una silla secretarial como mobiliario ideal.

Con los resultados obtenidos en el apartado del entorno virtual de aprendizaje se concluye que 92 alumnos cuentan con una buena iluminación en su área de estudio, 54 mencionan que a menudo, 73 a veces y 8 refieren no contar con iluminación suficiente y adecuada. Este factor de la iluminación interviene de manera directa en el estado de concentración y prevención de daños oculares. En el caso de los ruidos ajenos a la clase, 201 alumnos mencionan tener algún tipo de ruido de fondo al estar en áreas comunes del hogar en donde la familia, amigos, vecinos, dispositivos electrónicos, ruidos exteriores al hogar, intefieren en la escucha activa durante 
las sesiones virtuales; solamente 24 alumnos mencionaron que cuentan con espacios libres de ruidos que pudieran alterar su percepción y concentración en cada una de sus clases (figura 4).

Figura 4. Cantidad de estudiantes según su adecuación del espacio de estudio

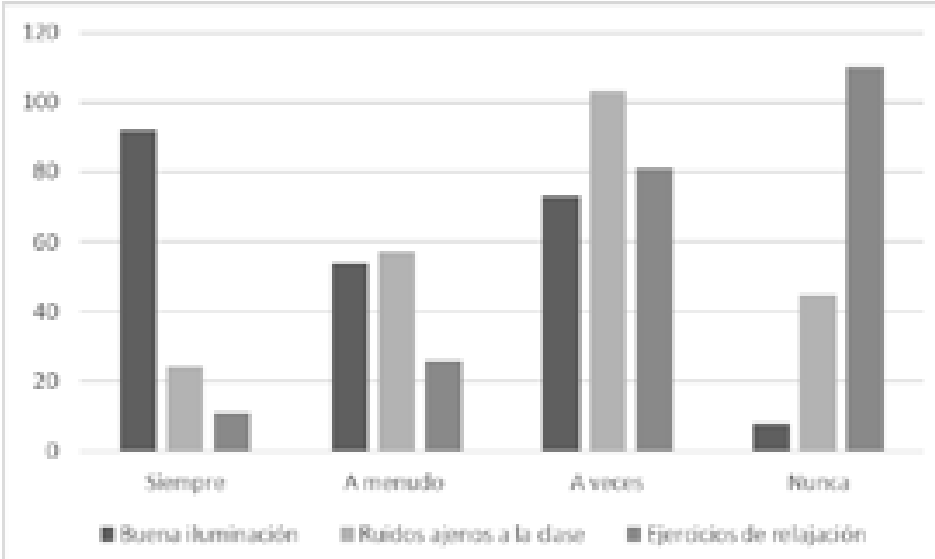

Nota: se puede apreciar en esta figura que más de $40 \%$ de los estudientes tienen una iluminación ideal; sólo $20 \%$ han logrado eliminar los ruidos ajenos a la clase; y sólo 4.8\% realizan los ejercicios de relajación de manera habitual.

La falta de aplicación de la ergonomía digital y el uso constante de los dispositivos electrónicos en las clases virtuales se manifiesta en cansancio y tensión muscular; para esto deben realizarse ejercicios de estiramiento muscular y articular con intensidad moderada, pero 110 alumnos mencionan que no realizan ningún tipo de ejercicios durante sus clases y 115 sí los realizan de manera constante.

En el caso de las plataformas de videoconferencias, más de $98 \%$ de los estudiantes se conectan al programa de Zoom, $0.4 \%$ en Google Meet y $0.9 \%$ a Facebook Live. La virtualidad en las actividades académicas genera también sentimientos de poco avance o aprendizaje al tener estereotipos negativos sobre la educación en línea. Es así que 81\% menciona tener afectaciones en su rendimiento escolar en comparación de cuando asistían a clases presenciales, y $18 \%$ refiere no haber tenido afectaciones en su rendimiento escolar. En el caso de las calificaciones que obtienen en las materias que se encuentran cursando de manera virtual, $70 \%$ de los estudiantes mencionan que se mantuvieron en el rango de lo esperado, $21 \%$ considera que las clases virtuales afectaron y disminuyeron los resultados de sus calificaciones y sólo $8 \%$ mencionan haber mejorado sus calificaciones en esta etapa de la educación virtual.

Dada la situación que los estudiantes presentan por el tipo de conexión a internet y disponibilidad de equipo tecnológico ideal, se tiene una gran variación en el número de horas que se conectan a las diferentes actividades académicas, como son las clases virtuales sincrónicas y las de extraclase: $1 \%$ se mantiene en conexión de 2 a 4 horas al día, $14 \%$ de 5 a 6 horas, $44 \%$ de 7 a 8 horas, $19 \%$ de 9 a 10 horas, $14 \%$ de 11 a 12 horas, $4 \%$ de 13 a 14 horas y $2 \%$ de 15 a 16 horas. 
La postura ideal que debe adoptar cada uno de los estudiantes de acuerdo con los lineamientos de la ergonomía digital, es estar sentado con la espalda apoyada al respaldo del asiento, los pies separados y firmes, la cabeza y los ojos tienen que estar a la misma altura de la pantalla o cámara del dispositivo, y los brazos descansados en el escritorio en un ángulo de 90 grados (véase figura 5).

Figura 5. Postura ideal para las clases virtuales en el hogar

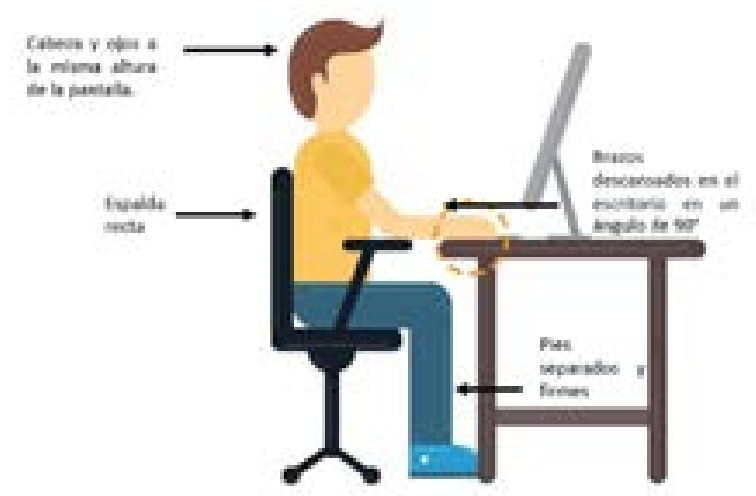

Nota: se puede apreciar en esta figura las recomendaciones para una postura ergonómica ideal en las clases virtuales. Imagen tomada de la Unidad Educativa Tomás Moro. https://www.tomasmoro.ec/manten-una-buena-postura-frente-al-computador/

Esta postura no la adoptan $78 \%$ de los estudiantes y solamente $21 \%$ sí conoce estos parámetros y los adopta en cada una de sus actividades virtuales. La no adopción de dichas posturas puede generar cansancio excesivo, dolores articulares y musculares y la sensación de somnolencia al no estar en la postura adecuada.

En el caso de los dispositivos electrónicos que se utilizan para la recepción y visualización de los contenidos virtuales de aprendizaje, tienen parámetros a seguir para que puedan ser óptimos y funcionales para los estudiantes. Los equipos de cómputo, como laptops y computadoras de escritorio, deben estar a una distancia de $60 \mathrm{~cm}$ de los ojos y los teléfonos celulares y tabletas a $35 \mathrm{~cm}$, para tener en condiciones óptimas la salud visual y postural. Se puede destacar que $8.2 \%$ de los alumnos coloca a una distancia ideal de $35 \mathrm{~cm}$ su dispositivo móvil o tableta y $16 \%$ de los que cuentan con equipos de cómputo lo coloca a $60 \mathrm{~cm}$ de distancia; esto refleja la falta de conocimientos sobre estos lineamientos de la ergonomía digital (figuras 6 y 7 ). 
Figura 6. Cantidad de estudiantes según la distancia a que se colocan los dispositivos móviles

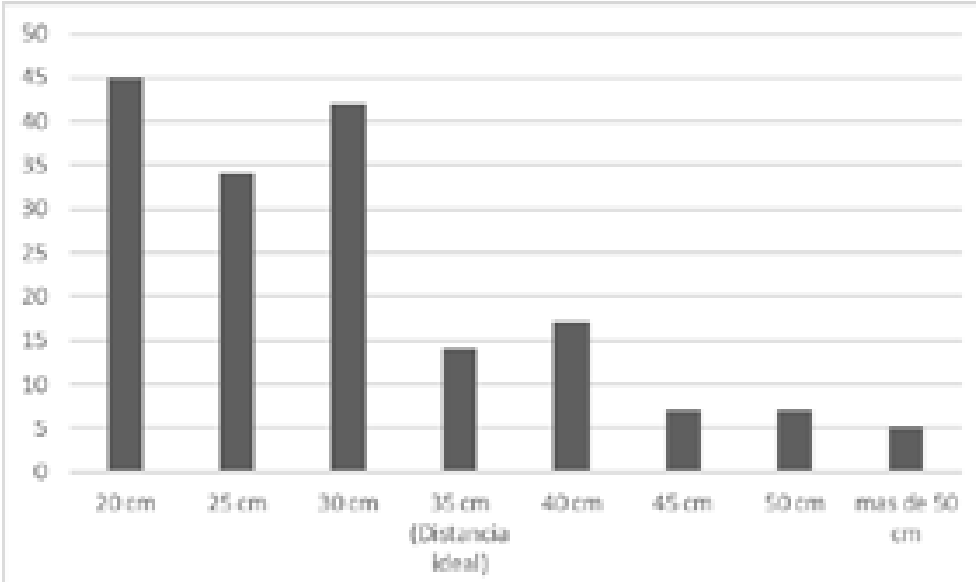

Nota: se aprecia que sólo 14 estudiantes mantienen la distancia ideal de 35cm entre sus dispositivos móviles.

Figura 7. Cantidad de estudiantes según la distancia a que se colocan de los equipos de cómputo

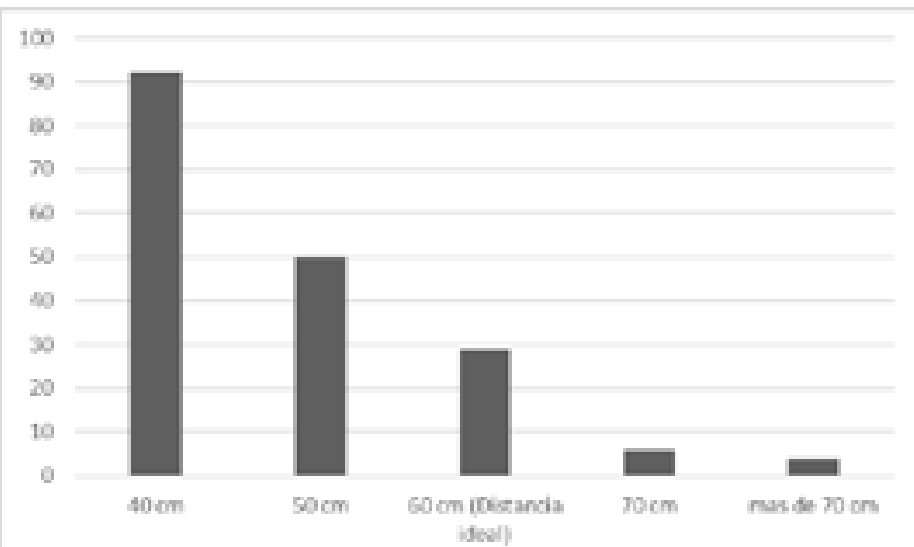

Nota: se aprecia que sólo 29 estudiantes mantienen la distancia ideal de $60 \mathrm{~cm}$ entre sus equipos de cómputo.

El uso de los dispositivos tecnológicos para el aprendizaje virtual requiere también un manejo adecuado en cuestiones de higiene: es necesario realizar una limpieza y desinfección de manera diaria para evitar la acumulación de microorganismos que pudieran afectar la salud. En este rubro, 72 estudiantes refieren limpiar todos los días los dispositivos utilizados, 72 lo hacen a menudo, 67 sólo a veces y 16 mencionan nunca haber limpiado sus equipos receptores. En el caso de las medidas para disminuir los agentes distractores como las notificaciones de los mensajes de texto (WhatsApp, Facebook), sólo 50 alumnos los mantienen desactivados en el transcurso de sus clases. 


\section{Discusión}

Los ambientes virtuales de aprendizaje son un elemento importante para el proceso de enseñanza-aprendizaje de los estudiantes del nivel superior, ya que permite desarrollar las actividades de manera flexible, dinámica, confortable y con medidas que mantienen la correcta postura de los estudiantes en los espacios designados por ellos en sus hogares. Martínez (2015) y Rodríguez (2017) mencionan que los factores que inciden en los Entornos Virtuales de Aprendizaje, como iluminación, ruido, notificaciones de los medios de comunicación móviles, la postura y la ergonomía digital, desempeñan un papel importante para facilitar el aprendizaje virtual a los estudiantes, pero que a la vez pueden ser factores que limiten este proceso.

De acuerdo con Contreras (2019), existen brechas en torno a la creación de un espacio ideal de aprendizaje virtual en el hogar, $\mathrm{y}$ al no contar con el espacio propio y adecuado para recibir las clases, la falta de equipos de cómputo o dispositivos móviles para las conexiones virtuales, o la falta de mobiliario adecuado para el desempeño en los espacios virtuales de aprendizaje en línea, se limitan el aprovechamiento y rendimiento académico escolar. De igual manera, García (2020) menciona que es imperativo contar con espacios adecuados con pocos distractores dentro del hogar. Con esto se pone de manifiesto que los estudiantes de la Escuela de Enfermería no cuentan con espacios adecuados y diseñados para poder recibir de manera óptima sus clases sincrónicas por medio de las salas de videoconferencias, al tener que recibirlas desde su cuarto individual, el patio, en el comedor o cocina y algunos en casa de otro familiar o en un ciber, y sólo muy pocos han logrado adecuar un espacio exclusivamente para sus clases.

El uso de las herramientas tecnológicas y digitales para las clases virtuales son necesarias ya que facilitan y acercan la universidad al hogar de cada uno de los estudiantes. Basantes (2017) menciona que el uso de computadoras o celulares tiene una gran funcionalidad en los ambientes virtuales de aprendizaje, y Sabido (2014) refiere que el uso particular de los celulares puede generar en los estudiantes de nivel superior una inquietud constante para utilizarlo para fines distintos, como la revisión de mensajes de texto, llamadas y redes sociales cuando están en las clases sincrónicas, que constituyen un fuerte distractor durante sus sesiones. En esta investigación se estima que $44 \%$ de los estudiantes disponen de celulares para recibir sus clases, teniendo con esto el riesgo de disminución del rendimiento escolar, y más de $50 \%$ sí cuentan con una computadora, que se considera la herramienta ideal para las actividades virtuales.

Castro (2015), en sus estudios realizados en la Universidad Nacional de Costa Rica sobre los espacios de estudio áulicos y de los hogares, afirma que la iluminación es un factor determinante en los grados de concentración y los niveles de fatiga física y mental, y sostiene también que hay una correlación estrecha del rendimiento dependiendo de si se tiene una iluminación natural o artificial. Que el ruido del ambiente, tanto interior como exterior del aula u hogar, afecta de igual manera en la concentración y recepción completa del mensaje que emiten los docentes en las plataformas digitales y repectuten en problemas de aprendizaje; para Mogas (2020) estos 
elementos que afectan al aprendizaje continuo y dinámico en los estudiantes, deben ser controlados para mitigar sus efectos a corto plazo en los resultados y pruebas de aprendizaje. En este contexto, se vislumbra que más de $59 \%$ de los estudiantes de esta escuela de enfermería no cuenta con una iluminación natural o artificial acorde a los requerimientos básicos, y más de 79\% refiere que también tiene ruido de fondo que no permite la recepción ideal del mensaje de cada clase. Lo anterior se combina con los datos obtenidos en el rubro del espacio destinado en el hogar para las clases virtuales, que resultan inadecuados, que tienen que compartir con los demás miembros del hogar, quienes con sus actividades diarias generan ruido de manera inevitable.

El hecho de estar sentado y conectado en las plataformas virtuales por largas horas del día genera cansancio físico que afecta a la estructura osteomioarticular del cuerpo al adoptar posturas que no son las correctas o ideales, por ende, también genera incomodidad en cada sesión virtual, de acuerdo a lo que sostiene Gómez (2017).

El tipo de mobiliario con el que cuentan los estudiantes de esta universidad no es el ideal para las largas horas de carga horaria académica, ya que casi todos trabajan sobre mesas y sillas de madera diseñadas para otros usos en el hogar, esto genera cansancio y desesperación al estar en espacios incomodos para las clases.

La distancia que se debe guardar para cada dispositivo tecnológico que se utilizado para las clases virtuales, como celulares y computadoras portátiles o de escritorio, debe ser dispuesto de acuerdo con lineamientos de la ergonomía digital para no dañar la vista y mejorar la recepción de los gráficos de cada pantalla o monitor. De acuerdo con Guilleumas (2010), lo ideal es que en el caso de dispositivos móviles y computadoras de escritorio se coloquen a 35 y 60 centímetros respectivamente, para mejorar la interacción con estos medios tecnológicos. Estos datos son desconocidos por una amplia población de estudiantes universitarios y pasan desapercibidos, exponiendo con esto que hace falta capacitación y conscientización para el uso correcto de los dispositivos tecnológicos.

Figura 8. Distancia ideal de los dispositivos electrónicos
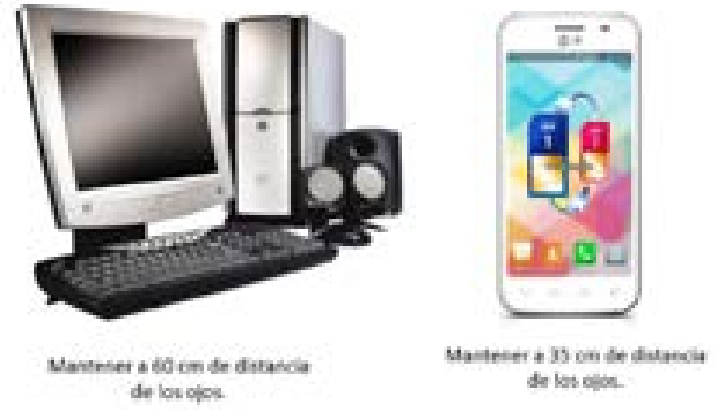

Nota: se aprecia la distancia ideal que se debe guardar en relación con la cabeza y ojos de los estudiantes. Fuente: tomado y modificado del libro Informática I (p. 9) de Francisco José Villazán Olivares, 2009. 
Al analizar los datos obtenidos a lo largo de este estudio, se puede concluir que el entorno virtual de aprendizaje y la aplicación de la ergonomía digital de los estudiantes de la Licenciatura en Enfermería de la Universidad Mesoamericana necesitan adecuarse para mejorar el proceso de aprendizaje y cuidar la salud física del educando en esta etapa de educación virtual provocada por las restricciones sanitarias que ha impuesto la pandemia por Covid-19.

\section{Referencias}

Basantes, A. (2017). Los dispositivos móviles en el proceso de aprendizaje de la Facultad de Educación, Ciencia y Tecnología de la Universidad Técnica del Norte de Ecuador. Revista de Formación Universitaria, 10(2), 79-88.

Calderón, M. (2020). Principales cifras del Sistema Educativo Nacional 2019-2020. México: SEP.

Castro, M. (2015). Los ambientes de aula que promueven el aprendizaje, desde la perspectiva de los niños y niñas escolares. Revista Electrónica Educare, 19(3), 1-32.

Chiecher, A. (2019). Estudiantes en contextos de educación a distancia. Variables vinculadas con el logro académico. Revista Iberoamericana de Educación a Distancia, 22(2), 1-20.

Contreras, A. (2019). Ambientes virtuales de aprendizaje: dificultades de uso en los estudiantes de cuarto grado de primaria. Revista de Trabajo Social e Intervención Social, (27), 215-240.

Fernández, E. (2017). Una mirada a los desafíos de la educación superior en México. Revista de Innovación Educativa, 17(74), 183-207.

García, M. (2020). La docencia desde el hogar: una alternativa necesaria en tiempo de COVID-19. Revista Polo del Conocimiento, 5(4), 304-324.

Gómez, C. (2017). El cuerpo como mediador de aprendizaje. México: Universidad Iberoamericana.

Gómez, F.; J. López (2019). Lesiones en docentes en educación física en Cataluña: análisis de la percepción ergonómica en su puesto de trabajo. Apuntes. Educación Física y Deportes, 35(135), 48-67.

Granados, J. (2019). Relación entre el uso del aula virtual y rendimiento académico en estudiantes del curso de Bioquímica para Enfermería de la Universidad de Costa Rica. Revista Educación, 43(2).

Guilleumas, R. (2010). TIC y educación, móviles en el aula de clases. Congreso lberoamericano de Educación. Metas 2021. Argentina. https://www.adeepra.org.ar/congresos/Congreso IBEROAMERICANO/TICEDUCACION/RLE2117Guile.pdf

Lara, R. (2020). Educación en enfermería en tiempos de pandemia: desafíos presentes y futuros. Ciencia y Enfermería. Revista Iberoamenricana de Investigación, 26, 1-3.

Laredo, A. (2020). Educación superior en México. España: ICEX España Exportación e Inversiones. 
Lloyd, M. (2020). Desigualdades educativas y la brecha digital en tiempos de COVID-19. En Casanova, H. (coord.). Educación y pandemia: una visión académica. México: UNAM, 115-121. http://132.248.192.241:8080/jspui/bitstream/IISUE UNAM/546/1/LloydM 2020 Desigualdades educativas.pdf

López, B.; A. Cuesta (2020). Higiene postural y ergonomía en el ámbito escolar: una perspectiva desde la fisioterapia. Revista de Estudios de Juventud, (79), 147-157. http://www.injuve.es/ sites/default/files/revista 79 9.pdf

López, M.; A. Estrada (2020). Desempeño académico de estudiantes en educación virtual. Algunos factores negativos. Revista de Educación e Investigación, 84-90. https://www3.azc.uam. $\mathrm{mx} /$ sieee/cuartoseminario/ponencias/ponencia10.pdf

Luengo-Martínez, C.; O. Sanhueza-Alvarado (2016). Formación del licenciado en Enfermería en América Latina. Revista Aquichan, 10(2), 240-255.

Moreno, J.; M. Rodríguez (2011). Capacidad productiva y educación superior en las entidades federativas de México. Revista Espacios Públicos, 13(31), 190-215.

Martínez, N.; E. Ruiz; R. Galindo (2015). Ambientes virtuales de aprendizaje y sus entornos con diseños abiertos y restringidos para la construcción del conocimiento; diferencias y similitudes. VI Congreso Virtual Iberamericano de Calidad en Educación Virtual y a Distancia, EduQ@. http://www.eduqa.net/eduqa2015/images/ponencias/eje1/1 aa Martinez Nadia Ruiz Edith Galindo Rosa Ambientes virtuales de aprendizaje y sus entornos con disenos abiertos y restringidos para la construccion del conocimiento diferencias y similitudes

Mendoza, J. (2018). Situación y retos de la cobertura del Sistema Educativo Nacional. Perfiles Educativos, 40 (especial). México: UNAM. https://doi.org/10.22201/iisue.24486167e.2018. Especial.59179

Mogas, J.; R. Palau; M. Márquez (2020). Acústica del aula: necesidad de las aulas inteligentes para solucionar los efectos de la contaminación sonora sobre el personal docente. Tecnologías Educativas y Estrategias Didácticas. España: Universidad de Málaga, 723-732.

Rivero, L.; R. Samino; E. Pérez (2008). Rendimiento académico y modelos virtuales de enseñanza universitaria en economía de la empresa. En Pindado, J.; G. Payne (coords.). Estableciendo puentes en una economía global (ponencias), 1, 35. España: Escuela Superior de Gestión Comercial y Marketing, (ESIC). https://dialnet.unirioja.es/servlet/articulo?codigo=2712303

Rodríguez, M.; H. Barragán (2017). Entornos virtuales de aprendizaje como apoyo a la enseñanza presencial para potenciar el proceso educativo. Revista Killkana Sociales, 01(02), 7-14.

Rodríguez, H.; M. Botero; L. Restrepo (2015). Factores relacionados con el uso de ambientes virtuales de aprendizaje (AVA) en la educación superior. Revista Virtual Universidad Católica del Norte, (46), 39-46. 
Sabido, M.; E. Martínez (2014). Uso del télefono celular como herramienta en las estrategias de aprendizaje. Memorias VIII Congreso Internacional de Innovación Educativa, 1-8. https:// www.repo-ciie.dfie.ipn.mx/pdf/687.pdf

Salcedo-Álvarez, R. (2017). El profesional de enfermería que México necesita. Revista de Enfermería del Instituto Mexicano del Seguro Social, 25(1), 75-79.

Sánchez, M.; C. Moreno; R. Córdova; M. Aguilar (2016). Ambientes virtuales de aprendizaje, como apoyo en la educación presencial. Reencuentro. Análisis de Problemas Universitarios, (72), 5570.

Torres, Á. (2002). Red UAM: grupos virtuales de aprendizaje colaborativo. Reencuentro. Análisis de Problemas Universitarios, (35), 38-48.

Zurita, C. (2020). Análisis critíco de ambientes virtuales de aprendizaje. Utopía y Praxis Latinoamericana, 25(11), 1-15. 